\title{
Ray-Tracing Analysis of Anisotropic Neutrino Radiation for Estimating Gravitational Waves in Core-Collapse Supernovae
}

\author{
Kei Kotake ${ }^{1}$, Wakana Iwakami ${ }^{2}$, Naofumi Ohnishi ${ }^{2}$, and Shoichi Yamada ${ }^{3,4}$ \\ ${ }^{1}$ Division of Theoretical Astronomy/Center for Computational Astrophysics, National \\ Astronomical Observatory of Japan, 2-21-1, Osawa, Mitaka, Tokyo, 181-8588, Japan \\ kkotake@th.nao.ac.jp \\ ${ }^{2}$ Department of Aerospace Engineering, Tohoku University, 6-6-01 Aramaki-Aza-Aoba, \\ Aoba-ku, Sendai, 980-8579, Japan \\ ${ }^{3}$ Science \& Engineering, Waseda University, 3-4-1 Okubo, Shinjuku, Tokyo, 169-8555, \\ Japan \\ ${ }^{4}$ Advanced Research Institute for Science and Engineering, Waseda University, 3-4-1 \\ Okubo, Shinjuku,Tokyo, 169-8555, Japan
}

\begin{abstract}
We propose a ray-tracing method to estimate gravitational waves (GWs) generated by anisotropic neutrino emission in supernova cores. To calculate the gravitational waveforms, we derive analytic formulae in a useful form, which are applicable also for three-dimensional computations. Pushed by evidence of slow rotation prior to core-collapse, we focus on asphericities in neutrino emission and matter motions outside the protoneutron star. Based on the two-dimensional (2D) models, which mimic SASI-aided neutrino heating explosions, we compute the neutrino anisotropies via the ray-tracing method in a post-processing manner and calculate the resulting waveforms. For simplicity, neutrino absorption and emission by free nucleons, dominant processes outside the PNSs, are only taken into account, while the neutrino scattering and the velocity-dependent terms in the transport equations are neglected. With these computations, it is found that the waveforms exhibit more variety in contrast to the ones previously estimated by the ray-by-ray analysis (e.g., Kotake et al. (2007)). In addition to a positively growing feature, which was predicted to determine the total wave amplitudes predominantly, the waveforms are shown to exhibit large negative growth for some epochs during the growth of SASI. These features are found to stem from the excess of neutrino emission in lateral directions, which can be precisely captured by the ray-tracing calculation. Reflecting the nature of SASI
\end{abstract}


which grows chaotically with time, there is little systematic dependence of the input neutrino luminosities on the maximum wave amplitudes. Due to the negative contributions and the neutrino absorptions appropriately taken into account by the ray-tracing method, the wave amplitudes become more than one-order-of magnitude smaller than the previous estimation, thus making their detections very hard for a galactic source. On the other hand, it is pointed out that the GW spectrum from matter motions have its peak near $\sim 100 \mathrm{~Hz}$, reflecting the SASI-induced matter overturns of $O(10) \mathrm{ms}$. Such a feature could be characteristic for the SASI-induced supernova explosions. The proposed ray-tracing method will be useful for the GW prediction in the first generation of 3D core-collapse supernova simulations that do not solve the angle-dependent neutrino transport equations as part of the numerical evolution.

Subject headings: supernovae: collapse — gravitational waves — neutrinos hydrodynamics

\section{Introduction}

No longer gravitational-wave astronomy is a fantasy. In fact, gravitational wave detectors, such as LIGO (Thorne 1995; Abbott et al. 2005), VIGRO1, GEO6002, TAMA300 (Ando \& the TAMA collaboration 2002; Ando et al. 2005), and AIGO3 with their international network of the observatories, are beginning to take data at sensitivities where astrophysical events are predicted (see, e.g., Hough et al. (2005) for a recent review). For the detectors, core-collapse supernovae especially in our Galaxy, have been proposed as one of the most plausible sources of gravitational waves (see, for example, Kotake et al. (2006); Ott (2009) for recent reviews). Since the gravitational waves (plus neutrinos) are the only tool that gives us the live information of the central engine of core-collapse supernovae, the detection is important not only for the direct confirmation of general relativity but also for disclosing the supernova physics itself.

Traditionally, most of the theoretical predictions of gravitational waves (GWs) have focused on the bounce signals (e.g., Zwerger \& Müller (1997); Kotake et al. (2003); Shibata \& Sekiguchi (2004); Ott et al. (2004); Obergaulinger et al. (2006); Cerdá-Durán et al. (2007); Dimmelmeier et al.

\footnotetext{
${ }^{1}$ http://www.ego-gw.it/

${ }^{2}$ http://geo600.aei.mpg.de/

${ }^{3}$ http://www.gravity.uwa.edu.au/
} 
(2007); Scheidegger et al. (2008); Dimmelmeier et al. (2008) and references therein). However recent stellar evolution calculations suggest that rapid rotation assumed in most of the previous studies is not canonical for the progenitors with neutron star formation (Heger et al. 2005). To explain the observed rotation periods of radio pulsars, the rotation periods of the iron core before collapse are estimated to be larger than $\sim 100 \mathrm{sec}$ (Ott et al. 2006). In such a slowly rotating case, the detection of the bounce signals becomes very hard even by the next-generation laser interferometers for a Galactic supernova (e.g., Kotake et al. (2004)).

Besides the rapid rotation of the core, convective matter motions and anisotropic neutrino emission in the much later postbounce phase are expected to be the primary GW sources with comparable amplitudes to the bounce signals. Thus far, various physical ingredients for producing asphericities and the resulting GWs in the postbounce phase, have been studied such as the roles of pre-collapse density inhomogeneities (Burrows \& Haves 1996; Müller \& Janka 1997; Fryer 2004; Frver et al. 2004), moderate rotation of the iron core (Müller et al. 2004), g-mode oscillations of protoneutron stars (PNSs) (Ott et al. 2006), and SASI (Kotake et al. 2007; Marek et al. 2009).

Among them, we focused on the GWs originated from the asphericities produced by the standing accretion shock instability (SASI) (Kotake et al. 2007). Here SASI, becoming very popular in current supernova researches, is a uni- and bipolar sloshing of the stalled supernova shock with pulsational strong expansion and contraction (see, e.g., Blondin et al. (2003); Scheck et al. (2004); Ohnishi et al. (2006); Foglizzo et al. (2007); Blondin \& Mezzacappa (2007); Iwakami et al. (2008a,b) and references therein). Based on the two-dimensional (2D) simulations, which demonstrate the neutrino-driven explosions aided by SASI using the lightbulb scheme (see Janka \& Müller (1996); Ohnishi et al. (2006) for details), it was pointed out that the GW amplitudes from anisotropic neutrino emission increase almost monotonically with time, which are dominant over the ones from matter motions, and that such signals may be visible to next-generation detectors for a Galactic source. More recently, Marek et al. (2009) analyzed the GW emission based on their long-term 2D Boltzmann (ray-by-ray) simulations, which seem very close to produce the SASI-aided neutrino-driven explosions (Marek \& Janka 2009). They also found that the GWs from neutrinos with continuously growing amplitudes (but with the different sign of the amplitudes in Kotake et al. (2007)), are dominant over the ones from matter motions. They proposed that the thirdgeneration class detectors such as the Einstein Telescope are required for detecting the GW signals with a good signal-to-noise ratio.

Except for the acoustic mechanism (Ott et al. 2006) and the magnetohydrodynamic mechanism (e.g., Yamada \& Sawai (2004); Kotake et al. (2004); Ardeljan et al. (2005); Shibata et al. (2006); Burrows et al. (2007); Takiwaki et al. (2009) and references therein), both of which 
produce strong mass-quadrupole GWs, all the studies in the postbounce phase mentioned above, rely basically on the conventional neutrino-heating mechanism (Bethe 1990). They agree that the GW amplitudes from anisotropic neutrino emission are dominant over the ones from mass motions. This means that accurate estimation of the neutrino anisotropy is indispensable for understanding the gravitational radiation from core-collapse supernovae, which requires to estimate precisely the directional dependence of the neutrino intensity emitted from the central cores. In the previous simulations with the light-bulb approximations (Müller \& Janka 1997; Kotake et al. 2007), there was no way but to estimate the angle-dependent neutrino luminosities, assuming that neutrinos are emitted purely radially in each lateral bin of the computational polar grid. This ray-by-ray treatment cannot capture the neutrino emission in the lateral directions entirely. The FLD(flux-limited-diffusion) schemes (either multi-energy (Walder et al. 2005) or single-energy FLD (Burrows \& Haves 1996; Fryer 2004; Fryer et al. 2004)) tend to smooth out the local and global neutrino anisotropies due to their diffusion characters. Any ray-by-ray transport schemes (Buras et al. 2003; Marek \& Janka 2009), albeit coupled to the Boltzmann transport and thus being one of the most sophisticated treatment at present, replaces the $2 \mathrm{D}$ neutrino transport with the 1D transport problem along radial rays in every lateral bin, leading to the overestimation of the directional dependence of the neutrino anisotropies (see discussions in Marek et al. (2009)). More recently, fully 2D multi-angle Boltzmann transport simulations become practicable (Ott et al. 2008), however too computationally expensive currently to perform the simulations, satisfying required number of the momentum-space angles in order to capture the neutrino anisotropies accurately (e.g., Ott (2009)).

These situations motivate us to propose a method to estimate the neutrino anisotropies, directly linked to the accurate estimation of the neutrino-originated gravitational radiation. Pushed by the striking evidences that support the slow rotation of iron cores (Heger et al. 2005; Ott et al. 2006), we consider an idealized situation that the neutrino radiation field from the PNSs are isotropic. This means that no GWs are assumed to be generated inside due to the isotropy. Then we focus on the asphericities outside the PNSs, which are produced by the growth of SASI. To this end, we utilize the 2D SASI models as in our previous paper (Kotake et al. 2007), in which the neutrino luminosities from the PNSs are adjusted to trigger explosions aided by SASI (Ohnishi et al. 2006). Since the regions outside the PNSs are basically optically thin to neutrinos, we solve the transport equations by making use of the ray-tracing (long-characteristics) method in a post-processing manner. For the sake of simplicity, we consider here only neutrino absorptions and emission by free nucleons, dominant processes outside the PNS, while the neutrino scattering and the velocity-dependent terms in the transport equation are neglected. We then study how the obtained gravitational waveforms could change from the ones obtained in the ray-by-ray analysis (e.g., Kotake et al. 
(2007)) and discuss their implications. Although the presented scheme is designed to be valid for the regions where neutrinos are thin to matter, we hope it to be useful for the GW prediction in the first generation of 3D core-collapse supernova simulations that do not solve the transport equations as part of the numerical evolution.

The plan of this paper is as follows. In section 2, first the formalism for calculating gravitational waveforms is described. Thereafter, we describe the ray-tracing method to calculate the direction-dependent neutrino luminosities. In Section 3, we shall briefly summarize the information how to construct the 2D exploding models, such as about the initial models and numerical methods. The main results are shown in Section 4 . We summarize our results and discuss their implications in Section 5 .

\section{Computing the Gravitational Wave Signatures}

\subsection{Formulae for Gravitational Waves from Anisotropic Neutrino Radiation}

To compute the gravitational waveforms from anisotropic neutrino radiation, we follow the formalism pioneeringly proposed by Epstein (1978). In the following, we present the formulae in a useful form, which is applicable also for $3 \mathrm{D}$ computations.

The two polarization states (of + and $\times$ modes) of GWs from anisotropic neutrino radiation satisfying the transverse-traceless conditions are given by Müller \& Janka (1997) as follows,

$$
h_{+}=\frac{2 G}{c^{4} R} \int_{0}^{t} d t^{\prime} \int_{4 \pi} d \Omega^{\prime}(1+\cos \theta) \cos 2 \phi \frac{d l_{\nu}\left(\Omega^{\prime}, t^{\prime}\right)}{d \Omega^{\prime}},
$$

and

$$
h_{\times}=\frac{2 G}{c^{4} R} \int_{0}^{t} d t^{\prime} \int_{4 \pi} d \Omega^{\prime}(1+\cos \theta) \sin 2 \phi \frac{d l_{\nu}\left(\Omega^{\prime}, t^{\prime}\right)}{d \Omega^{\prime}},
$$

where $G$ is the gravitational constant, $c$ is the speed of light, $R$ is the distance of the source to the observer,$d l_{\nu} / d \Omega$ represents the direction-dependent neutrino luminosity emitted per unit of solid angle into direction of $\Omega$. Variables with dash such as $\Omega^{\prime}$ represent the quantities

of the source coordinate system, while non-dashed ones belong to the the observer coordinate system (for the geometrical setup, see Figure 11). For convenience, we assume that $y$ axis coincides with $y^{\prime}$ axis and that the $z$-axis lies on the $\left(x^{\prime}, z^{\prime}\right)$ plane.

Using the following relations between the two coordinates,

$$
\begin{gathered}
\sin \theta \cos \phi=\sin \theta^{\prime} \cos \phi^{\prime} \cos \xi-\cos \theta^{\prime} \sin \xi, \\
\sin \theta \sin \phi=\sin \theta^{\prime} \sin \phi^{\prime},
\end{gathered}
$$




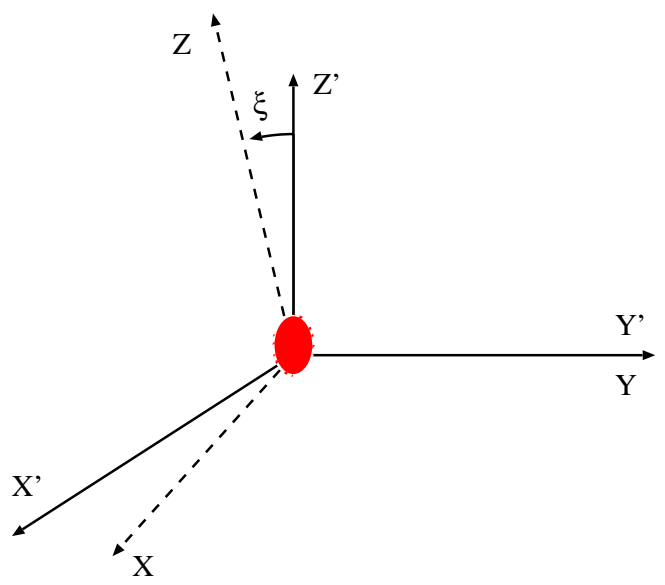

Fig. 1.- Source coordinate system $\left(x^{\prime}, y^{\prime}, z^{\prime}\right)$ and observer coordinate system $(x, y, z)$. The observer resides at the distant point on the $z$-axis. The viewing angle is denoted by $\xi$ which is the angle between $z$ and $z^{\prime}$ axis. The $z^{\prime}$ axis coincides with the symmetry axis of the source, presumably the rotational axis. Central red region illustrates the anisotropic neutrino radiation from a core-collapse supernova.

$$
\cos \theta=\sin \theta^{\prime} \cos \phi^{\prime} \sin \xi+\cos \theta^{\prime} \cos \xi
$$

$\theta$ and $\phi$ in equations (1, 2) are required to be expressed in terms of the angles $\theta^{\prime}, \phi^{\prime}$ with respect to the source coordinate valuables, and the viewing angle of $\xi$. After some algebra, one can readily derive the two modes of the GWs (equations (11, 2)) as follows,

$$
\begin{aligned}
h_{+}= & \frac{2 G}{c^{4} R} \int_{0}^{t} d t^{\prime} \int_{4 \pi} d \Omega^{\prime}\left(1+\sin \theta^{\prime} \cos \phi^{\prime} \sin \xi+\cos \theta^{\prime} \cos \xi\right) \times \\
& \frac{\left(\sin \theta^{\prime} \cos \phi^{\prime} \cos \xi-\cos \theta^{\prime} \sin \xi\right)^{2}-\sin ^{2} \theta^{\prime} \sin ^{2} \phi^{\prime}}{\left(\sin \theta^{\prime} \cos \phi^{\prime} \cos \xi-\cos \theta^{\prime} \sin \xi\right)^{2}+\sin ^{2} \theta^{\prime} \sin ^{2} \phi^{\prime}} \frac{\left.d \Omega^{\prime}, t^{\prime}\right)}{d \Omega^{\prime}}
\end{aligned}
$$

and

$$
\begin{aligned}
h_{\times}= & \frac{4 G}{c^{4} R} \int_{0}^{t} d t^{\prime} \int_{4 \pi} d \Omega^{\prime}\left(1+\sin \theta^{\prime} \cos \phi^{\prime} \sin \xi+\cos \theta^{\prime} \cos \xi\right) \times \\
& \frac{\sin \theta^{\prime} \sin \phi^{\prime}\left(\sin \theta^{\prime} \cos \phi^{\prime} \cos \xi-\cos \theta^{\prime} \sin \xi\right)}{\left(\sin \theta^{\prime} \cos \phi^{\prime} \cos \xi-\cos \theta^{\prime} \sin \xi\right)^{2}+\sin ^{2} \theta^{\prime} \sin ^{2} \phi^{\prime}} \frac{d l_{\nu}\left(\Omega^{\prime}, t^{\prime}\right)}{d \Omega^{\prime}}
\end{aligned}
$$

which will be useful in computing the GW signals also for 3D computations. It is noted that the sum of the squared amplitudes $\left|h_{+}\right|^{2}+\left|h_{\times}\right|^{2}$ is invariant under the rotation about the $z$-axis. To maximize the GW amplitudes in our 2D axisymmetric case, we assume that the observer is situated along the direction of the equatorial plane $(\xi=\pi / 2)$ as in Kotake et al. 


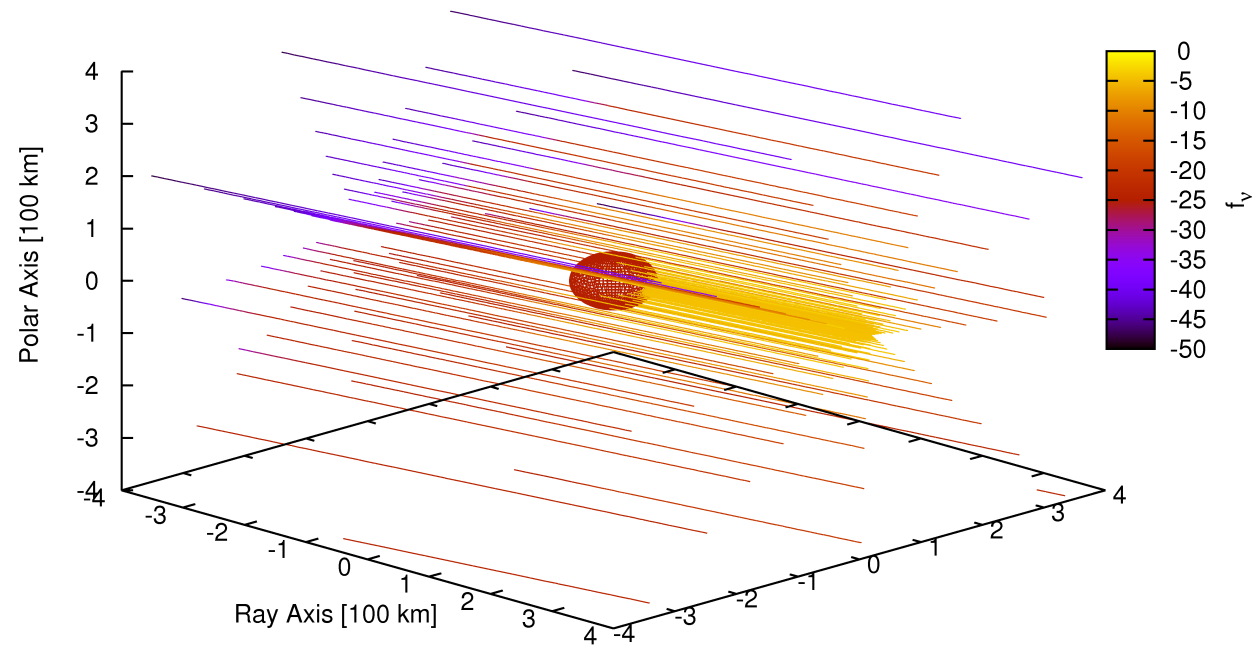

Fig. 2.- An example of ray-tracing of neutrinos for estimating $d l_{\nu}(\boldsymbol{\Omega}) / d \Omega$ towards a given direction of $\Omega$. The central region colored by red represents the surface of the protoneutron star (PNS) (located at $50 \mathrm{~km}$ in radius), which is the inner boundary of our computation. The color-scale on the rays shows the logarithmic values of $f_{\nu}$, the neutrino occupation probability (for $\epsilon_{\nu}=14 \mathrm{MeV}$ here), which is calculated by the line integral along each ray (equation (11)). For this snapshot taken from our 2D simulations, the higher values of $f_{\nu}$ are seen to come just in front of the PNS (yellow on the rays), while $f_{\nu}$ becomes smaller in the distant regions from the PNS. Doing ray-tracing calculations along every direction, $d l_{\nu}(\Omega) / d \Omega$ can be estimated through equation (14).

(2007). Then the only non-vanishing component is,

$$
\begin{aligned}
h_{+}^{\mathrm{e}} \equiv h_{\nu} & =\frac{2 G}{c^{4} R} \int_{0}^{t} d t^{\prime} \int_{4 \pi} d \Omega^{\prime}\left(1+\sin \theta^{\prime} \cos \phi^{\prime}\right) \frac{\cos ^{2} \theta^{\prime}-\sin ^{2} \theta^{\prime} \sin ^{2} \phi^{\prime}}{\cos ^{2} \theta^{\prime}+\sin ^{2} \theta^{\prime} \sin ^{2} \phi^{\prime}} \frac{d l_{\nu}\left(\theta^{\prime}, t^{\prime}\right)}{d \Omega^{\prime}} \\
& =\frac{4 G}{c^{4} R} \int_{0}^{t} d t^{\prime} \int_{0}^{\pi} d \theta^{\prime} \Phi\left(\theta^{\prime}\right) \frac{d l_{\nu}\left(\theta^{\prime}, t^{\prime}\right)}{d \Omega^{\prime}} .
\end{aligned}
$$

Here the subscripts of $\left(^{e}\right)$ and $\left({ }_{\nu}\right)$ indicates that the observer is situated in the equatorial plane and that the GWs are originated from neutrinos. $\Phi\left(\theta^{\prime}\right)$ depends on the angle measured from the symmetry axis $\left(\theta^{\prime}\right)$

$$
\Phi\left(\theta^{\prime}\right)=\pi \sin \theta^{\prime}\left(-1+2\left|\cos \theta^{\prime}\right|\right) .
$$


As given in Figure 1 of Kotake et al. (2007), this function has positive values in the north polar cap for $0 \leq \theta^{\prime} \leq 60^{\circ}$ and in the south polar cap for $120^{\circ} \leq \theta^{\prime} \leq 180^{\circ}$, but becomes negative values between $60^{\circ}<\theta^{\prime}<120^{\circ}$. In order to perform numerically the angular integration in equation (8) with high numerical accuracy, we recommend to perform it based on a Gaussian quadrature, because the function of $\Phi$ of equation (9) is not smooth near poles and equator. For estimating $h_{\nu}$ (equation (8)), we are yet to determine the directional dependent neutrino luminosities of $d l_{\nu} / d \Omega$, which we will estimate by the ray-tracing method in the next section.

Here it is noted that GWs generated by neutrinos are distinct from the ones from matter dynamics, because the former has the memory effect, which means that the gravitational amplitude jumps from zero to a nonvanishing value and it keeps the non-vanishing value even after the energy source of gravitational waves disappeared (see Braginskii \& Thorne (1987) for details). In equations (1,2), this nature can be directly seen as the time-integral. Other astrophysical emitters of GWs with memory have been elaborately studied for gamma-ray bursts (Hiramatsu et al. 2005; Suwa \& Murase 2009), Pop III stars (Suwa et al. 2007a,b), and inspiralling compact objects (Favata 2008) (see references therein).

As for the gravitational waves of the quadrupole radiation of mass motions, we em-

ploy the stress formula (see, e.g., equation (12) in Mönchmeyer et al. (1991)). In using the formula, we consider the self-gravity of matter in the accretion flow. In the following computations, we assume that the source is assumed to be located at our galactic center $(R=10 \mathrm{kpc})$ and also that the observer is situated in the direction of the equatorial plane.

\subsection{Ray-Tracing Calculations of Anisotropic Neutrino Luminosities}

Now we proceed to determine $d l_{\nu} / d \Omega$ in equation (8), the directional dependent neutrino luminosities through the ray-tracing method. Note in the following equations that we change dashed variables to non-dashed ones for the sake of simplicity.

In the ray-tracing approach, we consider transfer along the ray specified by a constant impact parameter $p$. The coordinate along $p$ is called $s$, satisfying

$$
r=\left(p^{2}+s^{2}\right)^{1 / 2}
$$

where $r$ is the radial coordinate. To estimate $d l_{\nu}(\Omega) / d \Omega$ along a given direction of $\Omega$, we draw rays of neutrinos as shown in Figure 2. As will be discussed soon later, in order to get the numerical convergence of $d l_{\nu}(\Omega) / d \Omega$, we need to set 45,000 rays for each direction, which consists of $500 \times 90$ rays, where the former is for the impact parameters covering from the inner- $\left(p_{\text {in }}=50 \mathrm{~km}\right)$ to the outer- boundary $\left(p_{\text {out }}=2000 \mathrm{~km}\right)$ of the computational domain 
and the latter is for covering the circumference (e.g., $2 \pi$ ) of the concentric circles on the plane perpendicular to the rays (see Figure 21). In the axisymmetric case here, we perform the ray-tracing calculations 60 times to cover the entire sphere, which is the number of the mesh points for the polar direction (e.g., section 3).

The transfer equation of the neutrino occupation probability $f_{\nu}\left(\epsilon_{\nu}, p, s\right)$ for a given neutrino energy $\epsilon_{\nu}$ along each ray is given by,

$$
\frac{d f_{\nu}\left(\epsilon_{\nu}, p, s\right)}{d s}=j\left(\epsilon_{\nu}, p, s\right)\left(1-f_{\nu}\left(\epsilon_{\nu}, p, s\right)\right)-\frac{f_{\nu}\left(\epsilon_{\nu}, p, s\right)}{\lambda}
$$

where $j$ and $\lambda$ is the emissivity and absorptivity via neutrino absorptions and emission by free nucleons $\left(\nu_{\mathrm{e}}+\mathrm{n} \rightleftarrows \mathrm{e}^{-}+\mathrm{p}\right)$ (Bruenn (1985); Ohnishi et al. (2006)), which are dominant processes outside the PNSs. The optical depth for those reactions are estimated by $\tau_{\nu}=$ $\int_{r}^{\infty} 1 / \lambda$. For the sake of simplicity, the neutrino scattering and the velocity-dependent terms in the transport equation are neglected here. Although $\bar{\nu}_{e}$ 's are taken into account the hydro simulations, we focus only on electron-type neutrinos here for simplicity, since they dominantly contribute to the resulting GWs outside the PNSs as will be discussed in section 4 (see also Kotake et al. (2007)). We use 16 neutrino energy bins which is logarithmically uniform and covers $0.9-110 \mathrm{MeV}$. Along each ray, $f_{\nu}$ is transferred by the line integral. When the line integral starts from the surface on the PNS (the lines coming from the PNS in Figure 2), we set the initial value of

$$
f\left(\epsilon_{\nu}\right)=\frac{1}{1+\exp \left(\epsilon_{\nu} / k_{\mathrm{B}} T_{\nu}\right)} \cdot \frac{1}{4 \pi},
$$

assuming that the neutrino distribution function at the surface is approximated by the FermiDirac distribution with a vanishing chemical potential. Here the neutrino temperature is set to be constant near $T_{\nu_{\mathrm{e}}}=4 \mathrm{MeV}$, whose values change slightly depending on the input neutrino luminosity. Note that these values are constant in time for each model. This is necessary to realize the steady unperturbed states (e.g., Ohnishi et al. (2006)). For the rays that do not hit the PNS, we start the line integral from the outer most boundary antipodal to the line of sight, where $f_{\nu}$ is essentially zero.

By a post process, we perform the line integral up to the outer-most boundary for each hydro-timestep. The time sampling for the postprocessing is about $\sim 1 \mathrm{~ms}$, which is sufficient here because the waveforms from neutrinos show much slower temporal variation ( $\gtrsim 50 \mathrm{~ms}$ ) as we will discuss in section 4. Since the regions outside the PNSs are basically thin to neutrinos (e.g., section 4.1), the transport equation is suited to be solved by making use of the ray-tracing (long-characteristics) method. For all the rays, our treatment in equation (11) is identical to neglect the time-retardation, which is equal to the light traveling time of neutrinos from the PNS to the outer boundary. So the difference of the light traveling 
time comes from the difference in the position of the neutrino emitting regions, which are close to the PNS surface of $\sim 50 \mathrm{~km}$. Divided by the speed of light, the difference of the time-retardation, is less than the order of $0.1 \mathrm{~ms}$, which is negligible for our computation, because the hydrodynamical timescale is longer than $\sim 10 \mathrm{~ms}$.

With $f\left(\epsilon_{\nu}, p, s_{\text {out }}\right)$, which is obtained by the line integral up to the outer-most boundary, the neutrino energy fluxes along a specified direction of $\boldsymbol{\Omega}$ can be estimated,

$$
\frac{d l_{\nu}(\Omega, p)}{d \Omega d S}=\int f\left(\epsilon_{\nu}, p, s_{\text {out }}\right) \cdot\left(c \epsilon_{\nu}\right) \cdot \frac{\epsilon_{\nu}^{2} d \epsilon_{\nu}}{(2 \pi \hbar c)^{3}} .
$$

By summing up the energy fluxes with the weight of the area in the plane perpendicular to the rays, we can find $d l_{\nu} / d \Omega$ along a specified direction $\Omega$,

$$
\frac{d l_{\nu}(\boldsymbol{\Omega})}{d \Omega}=\int \frac{d l_{\nu}(\boldsymbol{\Omega}, p)}{d \Omega d S} d S=\int_{p_{\text {in }}}^{p_{\text {out }}} d p 2 \pi p \frac{d l_{\nu}(\boldsymbol{\Omega}, p)}{d \Omega d S}
$$

Repeating the above procedures, $d l_{\nu}(\Omega) / d \Omega$ can be estimated for all the directions.

To verify the newly developed numerical code, we calculate $d l_{\nu}(\Omega) / d \Omega$ in a spherical medium as a test calculation. The left panel of Figure 3 shows how the deviation of $d l_{\nu}(\Omega) / d \Omega$ from its (polar)angle-averaged value, indicated by $\delta\left(d l_{\nu} / d \Omega\right) \equiv d l_{\nu} / d \Omega-\left(d l_{\nu} / d \Omega\right)_{\text {average }}$ in the figure, changes with the number of the neutrino rays. With increasing the number of the rays, the deviation is shown to be smaller especially near the polar regions (near $0^{\circ}, 180^{\circ}$ ), while the deviation near the equator (near $90^{\circ}$ ) is nearly converged, but with relatively larger errors than for the other direction. This is because the angular grid size near the equatorial belt becomes larger for the spherical coordinate system taken in our simulation. Right panel of Figure 3 shows the resulting GW amplitudes for the different number of the rays. It is noted that the amplitudes vanish formally for the isotropic configuration. From the restriction of the computational time, we choose to set 45,000 rays for our actual raytracing calculation (green circle in the right panel). With this choice, the numerical errors are suppressed to be an order of $10^{-27}$, which is typically $4-5$ orders of magnitudes smaller than typical GW amplitudes obtained in the models computed here. We have also checked the numerical convergence for a deformed matter configuration (see Figure 4).

\section{Construction of 2D Exploding Models}

As in Kotake et al. (2007), we assume that the explosion is powered by the neutrinoenergy deposition between the PNS and the standing accretion shock, probably one of the most promising way to blow up massive stars (e.g., Marek \& Janka (2009)). To trigger the 



Fig. 3.- Numerical tests, in which the ray-tracing calculation is done for a spherical medium using data at $t=294 \mathrm{~ms}$ for model A. Left panel shows the deviation of $d l_{\nu}(\Omega) / d \Omega$ from its angle-averaged value as a function of the angle measured from the polar axis for different number of the neutrino rays $\left(: N_{\text {ray }}\right)$ (see text for more details). Right panel shows the resulting GW amplitudes, which should formally vanish. The green circle represents for $N_{\text {ray }}=45,000$, which we employ for our actual ray-tracing calculation. Note in the right panel that the supernova is assumed to be located at a distance of $10 \mathrm{kpc}$.

explosions, we employ the so-called light-bulb approximation (see Janka \& Müller (1996); Ohnishi et al. (2006) for details) and adjust the neutrino luminosities from the PNSs, which was found to work well in 2D, capturing the main features obtained by more realistic simulations (e.g., Scheck et al. (2004, 2006)). It should be noted again that we focus on the GWs generated by the anisotropies associated with the growth of SASI, which develops outside the PNS. Therefore, the oscillations of the PNSs (Burrows et al. 2006) and the resulting efficient gravitational emission (Ott et al. 2006) or the enhanced neutrino emission near the equator of the PNS observed in Marek et al. (2009), cannot be treated in principle here.

Our hydrodynamic code is based on the ZEUS (Stone \& Norman 1992) and major modifications for the supernova simulations are already described in Kotake et al. (2003); Ohnishi et al. (2006). The computational grid is comprised of 300 logarithmically spaced radial zones to cover from the absorbing inner boundary of $\sim 50 \mathrm{~km}$ to the outer boundary of $2000 \mathrm{~km}$, and 60 polar $(\theta)$ uniform mesh points (see for the resolution tests in Kotake et al. $(2007))$.

The initial condition is a spherically symmetric steady accretion flow through a stalled shock wave to a PNS (Yamasaki \& Yamada 2005), which is also provided in the same manner of Ohnishi et al. (2006). In constructing the initial conditions, we assume a fixed density $\rho_{\text {in }}=10^{11} \mathrm{~g} \mathrm{~cm}^{-3}$ at the inner boundary. And the initial mass accretion rates and the initial 


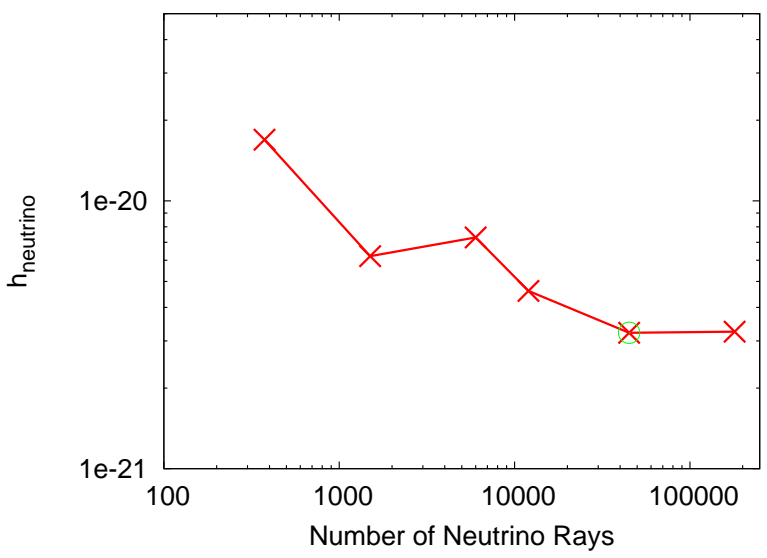

Fig. 4. - Same as the right panel of Figure 3 but for a deformed medium. Here we artificially impose a global quadrupole deformation $(\ell=2)$ to the matter distribution. It shows the numerical convergence of the GW amplitudes for $N_{\text {ray }}=45,000$.

mass of the central object are set to be $\dot{M}=1 M_{\odot} \mathrm{s}^{-1}$ and $M_{\text {in }}=1.4 M_{\odot}$, respectively. As in Kotake et al. (2007), the self-gravity of matter in the accretion flow is ignored. To induce non-spherical instability, we add random velocity perturbations of less than $1 \%$ of the unperturbed velocity. At the outer boundary, we adopt the fixed boundary condition consistent with the initial condition. On the other hand, the absorbing boundary is used at the inner boundary. We assume that the neutrino flux from the PNSs can be approximated by black-body emission.

Pushed by the evidences that the SASI-induced explosions are favorable for explaining the observed quantities such as the synthesized elements of SN1987A (Kifonidis et al. 2006), the pulsar kicks (Scheck et al. 2004) and spins (Blondin \& Mezzacappa 2007; Iwakami et al. 2008b), we mainly focus on the gravitational radiation in the models tuned to produce explosions. By changing the electron neutrino luminosity at the surface of the PNS in the range of $L_{\nu_{e}}=6.4-6.8 \times 10^{52}$ (10 foe) $\mathrm{erg} \mathrm{s}^{-1}$, we construct three exploding and one nonexploding models (see Table 1). Except for model D, we can observe the continuous increase of the average shock radius with the growth of SASI, reaching the outer boundary of the computational domain with the explosion energy of $\sim 10^{51} \mathrm{erg}$. Until this moment, we run the simulations (e.g., $\Delta t$ in the table), while in model $\mathrm{D}$, we terminated the simulation at about $800 \mathrm{~ms}$, not seeing the increase of the shock radius. 


\section{Results}

The left panel of Figure 5 shows the GW amplitudes contributed from anisotropic neutrino emission for different luminosity models. Comparing the right panel, which shows the total amplitudes (neutrino + matter), it can be seen that the gross structures of the waveforms are predominantly determined by the neutrino-originated GWs with the slower temporal variations ( $\gtrsim 50 \mathrm{~ms}$ ), to which the GWs from matter motions with rapid temporal variations $(\lesssim 10 \mathrm{~ms})$ are superimposed.

On the other hand, the waveforms shown in the left panel of Figure 6 are estimated simply by summing up the local neutrino cooling rates outside the PNSs with the ray-by-ray assumptions (Kotake et al. 2007) (see also Figure 2 in the paper). They are so much different from the ones obtained here. In contrast to the monotonic increase of the amplitudes with time, the waveforms here exhibit more variety, showing large negative growth at some epochs. And the GW amplitudes from neutrinos become more than one-order-of magnitude smaller than the previous estimation (compare Figures 5 and the left panel of 6 noting the different vertical scales). On the other hand, similarities between them are that the waveforms from neutrinos have generally a positively growing feature with time, and also that the electrontype neutrinos dominantly contribute to the wave amplitudes than for the anti-electron type neutrinos (right panel of Figure 6, see also Figure 3 in Kotake et al. (2007)). In the following, we first analyze this positive trend based on the ray-tracing calculations. And in section 4.2 , we clarify the reason for the negative growth, contributing to make the amplitudes much smaller than previously estimated.

Table 1. Model Summary

\begin{tabular}{cccccc}
\hline \hline Model & $L_{\nu_{e}}\left(10^{52} \mathrm{erg} / \mathrm{s}\right)$ & $\Delta t(\mathrm{~ms})$ & $h_{\nu, \mathrm{fin}}\left(10^{-22}\right)$ & $\left|h_{\text {tot }, \max }\right|\left(10^{-22}\right)$ & $E_{\mathrm{GW}, \nu}\left(10^{-12} M_{\odot} c^{2}\right)$ \\
\hline $\mathrm{A}$ & 6.8 & 509 & 8.7 & 7.7 & 0.44 \\
$\mathrm{~B}$ & 6.7 & 570 & 2.2 & 9.1 & 1.32 \\
$\mathrm{C}$ & 6.6 & 740 & 6.1 & 8.0 & 1.39 \\
$\mathrm{D}$ & 6.4 & 800 & 4.8 & 6.1 & 0.49 \\
\hline
\end{tabular}

Note. $-L_{\nu_{e}}$ denotes the input luminosity. $\Delta t$ represents the simulation time. $h_{\nu, \text { fin }}$ and $h_{\text {tot, } \max }$ represents the amplitudes of the neutrino-originated GWs at the end of the simulations and the maximum amplitudes (neutrino + matter) during the simulation time. $E_{\mathrm{GW}, \nu}$ is the radiated energy in the form of the neutrino GWs in unit of $M_{\odot} c^{2}$. Note that the supernova is assumed to be located at a distance of 10 kpc. 

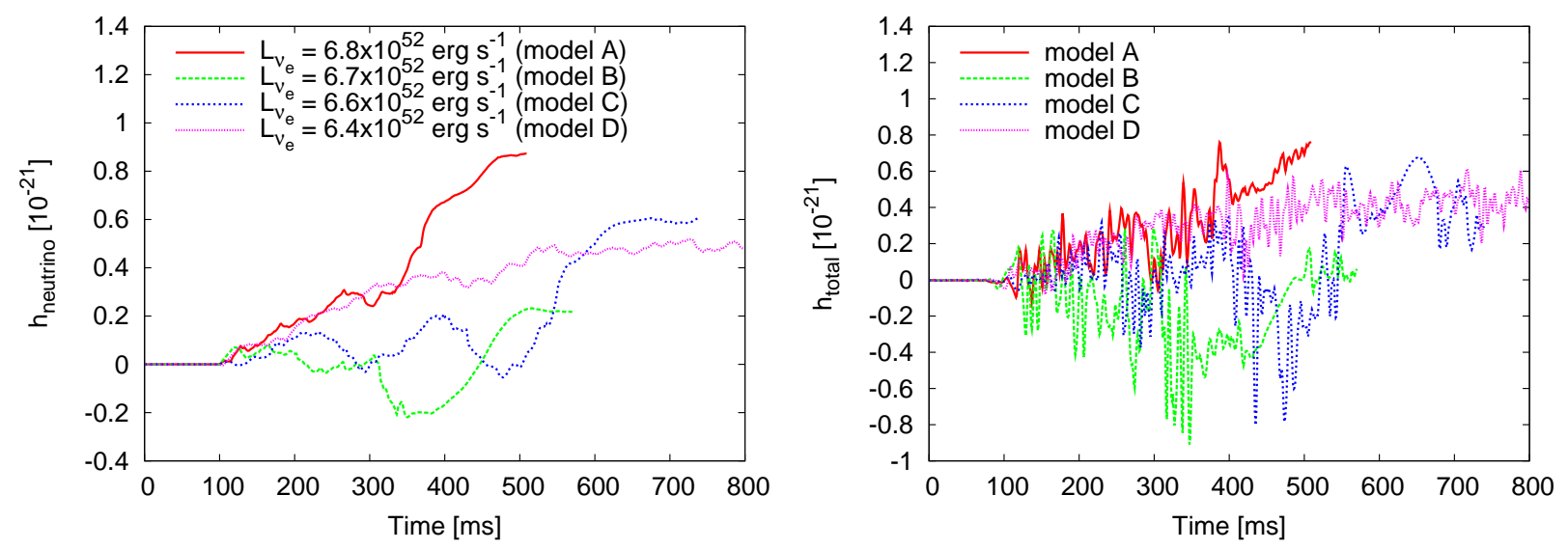

Fig. 5.- Gravitational waveforms only from anisotropic neutrino emission (left) and from the sum of neutrinos and matter motions (right). The time is measured from the epoch when the neutrino luminosity is injected from the surface of the neutrino sphere. In all the computed models, SASI enters to the non-linear regime at about $100 \mathrm{~ms}$, simultaneously making the amplitudes deviate from zero. Note that the supernova is assumed to be located at the distance of $10 \mathrm{kpc}$.

\subsection{Positively growing features}

To see the positively growing features clearly, we choose model A as a reference. The left panel of Figure 7 shows $d l_{\nu} / d \Omega$ in the vicinity of the north pole $(\theta=0)$, the equator $(\theta=\pi / 2)$, and the south pole $(\theta=\pi)$. In the following, we focus on the two epochs of $t$ $=294$ and $370 \mathrm{~ms}$, when the neutrino emission in the south pole dominate over the ones in the north pole and vice versa. The right panel of Figure 7 shows that the dominance of the neutrino emission in the north and south poles are closely anti-correlated. This is the consequence of the low-mode nature of SASI, here of $\ell=1$. In fact, the right panel of Figure 8 shows that at $294 \mathrm{~ms}$, the blob encompassing the regions inside the stalled shock is moving from the southern to the northern hemisphere, leading to the compression of the matter in the south hemisphere, which is vice versa at $370 \mathrm{~ms}$ (right panel).

Figure 9 shows various properties obtained by the ray-tracing calculation for $t=294$ ms. Top, middle, and bottom panel, is the case seen from the northern hemisphere, the equator, and the south hemisphere, respectively. Comparing the right panels, we can see that the rays with highest $f_{\nu}$, come from the high-temperature regions near the vicinity of the south pole (bottom right panel). As mentioned above, this is due to the compression of matter near the south pole, produced by the sloshing SASI, moving from the south to the northern hemisphere. 

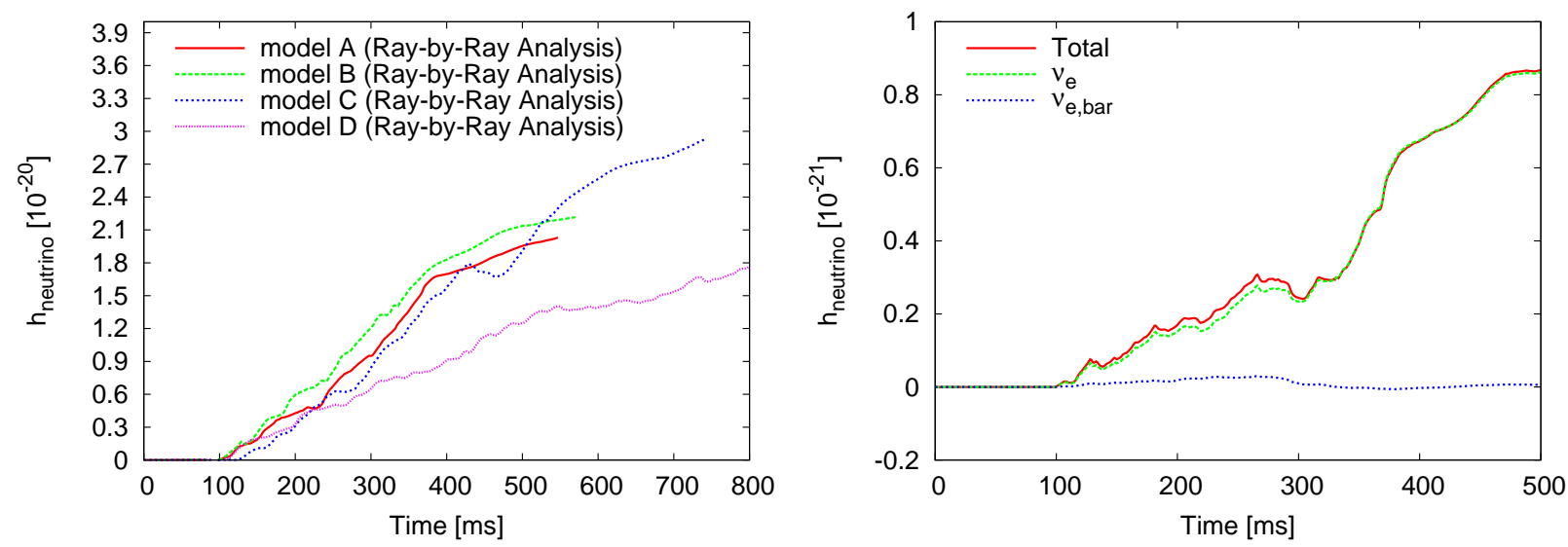

Fig. 6.- Gravitational waveforms from anisotropic neutrino emission estimated by the rayby-ray analysis of Kotake et al. (2007), showing a monotonic increase of the amplitudes with time (left). Right panel is the same as the left panel of Figure 5 for model A but with the contribution of anti-electron type neutrinos $\left(\bar{\nu}_{e}\right)$ to the waveform (indicated by "Total"), showing that the dominant contribution to the GW amplitudes comes from electron-type neutrinos $\left(\nu_{e}\right)$. The supernova is assumed to be located at the distance of $10 \mathrm{kpc}$.

In the left panels of Figure 9, it is noted that due to the axisymmetry, the distributions of the neutrino energy fluxes seen from the north (top left) and south hemispheres (bottom left) have the circumferential structures. The images of the energy fluxes may seem like an annular eclipse, which we explain as follows. Here for clarity, let's consider two rays of A and $\mathrm{B}$. The ray $\mathrm{A}$ is coming a point from $(X=0, Y=0)$ in the middle left panel, which in the middle right panel, is equal to the point along the line perpendicularly threading the center of the colored plane, but is positioned at $50 \mathrm{~km}$ away from the plane to the observer (:right direction in the panel). The ray B is coming from a point from $(X=0, Y \approx-50 \mathrm{~km})$ in the middle left panel, which is equal to the point, near the southern point on the colored plane in the middle right. The ray B travels to the observer, experiencing longer the hightemperature regions near the surface of the PNS. This makes the eclipse-like shining near the edge of the PNS. The left panel of Figure 10 is an enlargement of the bottom right panel of Figure 9 near the PNS, which clearly shows the rim-shining near $X=50 \mathrm{~km}$ (compare the right-hand side with the bottom left panel of Figure 91). The right-hand side in the right panel of Figure 10 depicts the optical depth of neutrinos at $\epsilon_{\nu}=14 \mathrm{MeV}$. It is seen that the high values of $f_{\nu}$ come from the higher temperature regions in the vicinity of the southern part of the PNS (left panel), where are thin to the neutrinos with the optical depth being smaller than $2 / 3$ (right panel). Such an optically thinness is favorable for the ray-tracing calculation here as discussed in section 2.2. 

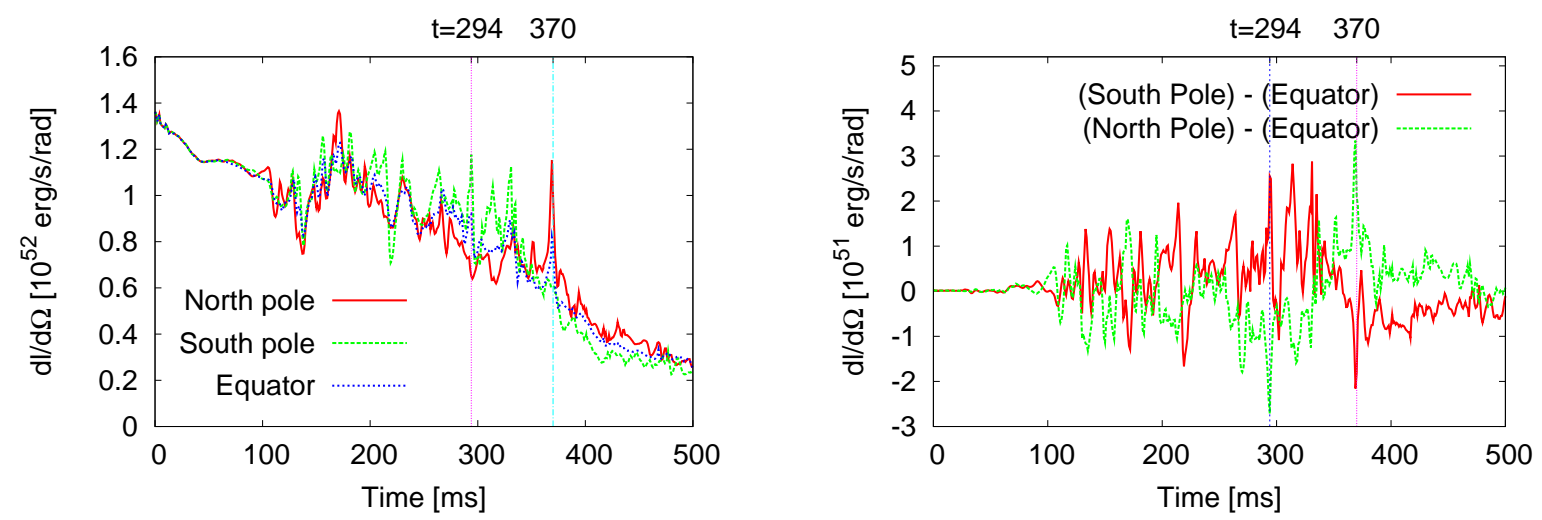

Fig. 7.- The directional dependent neutrino luminosity: $d l_{\nu} / d \Omega$ for model $\mathrm{A}$, in the vicinity of the north pole, the equator, and the south pole (left panel), and their differences from the equator (right panel). Vertical lines represent the epochs of $t=294$ and $370 \mathrm{~ms}$, respectively (see text for details).

Among the left panels of Figure 9, the highest values of the local neutrino energy fluxes $\left(: d l_{\nu} /(d \Omega d S)\right)$ come from the brightly shining southern poles seen from the equator (middle panel). However by summing up them with the weight of the area (e.g., $d S$ in equation (14) ), $d l_{\nu} / d \Omega$ are shown to become largest seen from the southern hemisphere (the red line in the left panel of Figure 11). The similar argument is true for $t=370 \mathrm{~ms}$ (Figure 12), while the neutrino luminosities seen from the northern hemisphere are higher than the ones from the southern hemisphere (the green line in the left panel of Figure 11). Noting again that $\Phi\left(\theta^{\prime}\right)$ in equation (8) are positive near the north and south polar caps, the dominance of the anisotropic neutrino luminosities in the vicinity near the north and south poles, makes the positively growing feature in the resulting GWs.

Figure 13 shows time evolution of a neutrino anisotropy parameter defined in Müller \& Janka (1997),

$$
\alpha(t)=\frac{1}{L_{\nu}(t)} \int_{4 \pi} d \Omega^{\prime} \Phi\left(\theta^{\prime}\right) \frac{d l_{\nu}\left(\Omega^{\prime}, t^{\prime}\right)}{d \Omega^{\prime}}
$$

where $L_{\nu}(t)$ is the total neutrino luminosity $L_{\nu}(t)=\int_{4 \pi} d \Omega^{\prime} d l_{\nu}\left(\Omega^{\prime}, t^{\prime}\right) / d \Omega^{\prime}$. This quantity is useful to see how the temporal changes of the neutrino anisotropy have impacts on the GW amplitudes. Either for models A or B, it can be seen that $\alpha$ keeps positive value with time in the later phase ( $\gtrsim 400 \mathrm{~ms}$ ). This coincides with the epoch when the low-modes explosion is triggered by SASI along the symmetry axis, which is also helpful for understanding the reason of the positive growth (compare the left panel of Figure 5). 



Fig. 8.- Temperature- (the left-half of each panel) and density- (the right-half) distributions in the meridian section for model A. The insert of each panel shows the neutrino GW amplitudes, in which the green point indicates the time of the snapshot. The central region colored by white $(50 \mathrm{~km}$ in radius) represents the inner boundary of our computation.

\subsection{Negatively growing features}

Now we move on to discuss large negative growth of the GW amplitudes seen in the waveforms (e.g., models $\mathrm{B}$ and $\mathrm{C}$ in Figure 5), which is newly found by the ray-tracing calculation (compare Figure 2 of Kotake et al. (2007)). Here we choose a snapshot at $t=330$ ms of model B as a reference, which shows the steep negative growth (see the insert of Figure 14).

The middle panel of Figure 15 shows that the neutrino emission seen from the equatorial plane are much stronger than the ones seen from the northern/southern hemispheres. The bright belt-like structure in the middle panel is from the high-temperature regions in the northern hemisphere, which are by chance surrounded by relatively low density (see cusp-like structure in the northern hemisphere of Figure 14 and 16). In this case, the area-weighted energy fluxes become larger seen from the equatorial plane (see right panel of Figure 11). In combination with the negative values of $\Phi\left(\theta^{\prime}\right)$ in equation (8) in the vicinity of the equatorial belts, this makes the negative growth in the GW amplitudes. Large negative amplitudes seen for some other epochs in other model such as model $\mathrm{C}$ (left panel of Figure 5) are also from the same reason. Such a feature is genuine outcome of the neutrino emission in the lateral direction, which is able to be captured correctly by the ray-tracing calculation. 
It is noted that the appearance of the negative growth has no systematic dependence of the input luminosities. In fact, as seen from Figure 5, the negative growth is observed for the intermediate luminosities models (models B and C), but not for the highest (model A) and smallest luminosity models (model D) (see also $\left|h_{\text {tot,max }}\right|$ in Table 1 ). This should reflect the nature of the SASI which grows chaotically and non-locally. Albeit with the negative growth, our results suggest that the positively growing features dominate over the negatively ones for the 2D models (see $h_{\nu \text {,fin }}$ in Table1). This is due to the axial symmetry, along which the SASI develops preferentially and the resulting anisotropies become larger.

As mentioned earlier, the neutrino GWs become more than one-order-of magnitude smaller than the previous estimation (compare $E_{\mathrm{GW}, \nu}$ in Table 1 and the one in Kotake et al. (2007)). This stems not only from the incursion of the negative contributions but also from the appropriate estimation of the neutrino absorptions made possible by the ray-tracing method. Previously the neutrino luminosity was estimated simply by summing up the local neutrino cooling rates outside the PNSs (Kotake et al. 2007), which fails to take into account the neutrino absorption correctly $(\lambda$ in equation (11) $)$. These two factors make the amplitudes much smaller than the previous estimation. As a result, the neutrino GWs, albeit dominant over the matter GWs in the lower frequencies below $\sim 10 \mathrm{~Hz}$ (Figure 17), become very difficult to be detected for ground-based detectors whose sensitivity is limited mainly by

the seismic noises at such lower frequencies (Ando \& the TAMA collaboration 2002; Thorne 1995; Weinstein 2002; LCGT Collaboration 1999).

On the other hand, the GWs from matter motions seem marginally within the detection limits of the currently running detector of the first LIGO and the detection seems more feasible for the detectors in the next generation such as LCGT and the advanced LIGO for a Galactic supernova. The spectra of the matter GWs have double peaks namely near $100 \mathrm{~Hz}$ and $1 \mathrm{kHz}$. While the latter comes from the rapidly varying local hydrodynamical instabilities with milliseconds timescales, the former is associated with the longer-term overturns of $O(10)$ ms induced by $\ell=2$ mode of SASI (see e.g., Figure 5 in Kotake et al. (2007)). These gross properties in the GW spectra are common to the other luminosity models. Thus the peak in the spectra near $\sim 100 \mathrm{~Hz}$ is found to be a characteristic feature obtained in the $2 \mathrm{D}$ models computed here.

\section{Summary and Discussion}

We proposed a ray-tracing method to estimate gravitational waves (GWs) generated by anisotropic neutrino emission in supernova cores. To calculate the waveforms, we derived the GW formulae in a useful form, which are applicable also for 3D computations. Pushed by the 
studies supporting the slow rotation prior to core-collapse, we considered an idealized situation that the neutrino radiation field from the protoneutron stars (PNSs) are isotropic. Then we focused on the asphericities outside the protoneutron stars, which are produced by the growth of standing accretion shock instability (SASI). Since the regions outside the PNSs are basically thin to neutrinos, we solve the transport equations by making use of the ray-tracing method in a post-processing manner. For simplicity, neutrino absorption and emission by free nucleons, dominant processes outside the PNSs, were only taken into account, while the neutrino scattering and the velocity-dependent terms in the transport equations were neglected. Based on the two-dimensional models, which mimic SASI-aided neutrino heating explosions, we estimated the neutrino anisotropies by the ray-tracing method and calculated the resulting GWs.

Our results show that the waveforms from neutrinos exhibit more variety in contrast to the ones previously estimated by ray-by-ray analysis (e.g., Kotake et al. (2007)). In addition to the positively growing feature, which was predicted to determine the total wave amplitudes predominantly, the waveforms show large negative growth for some epochs during the growth of SASI. Such a feature is a genuine outcome of the neutrino emission in lateral directions, which can be captured correctly by the ray-tracing calculation. Reflecting the nature of SASI which grows chaotically with time, little systematic dependence of the input neutrino luminosities on the maximum amplitudes and on the radiated GW energies are found. Due to the negative contributions and the neutrino absorptions appropriately taken into account by the ray-tracing method, the GWs from neutrinos become more than one-order-of magnitude smaller than the previous estimation, making their detections very hard for a galactic source. On the other hand, we point out that the gravitational-wave spectrum from matter motions have its peak near $\sim 100 \mathrm{~Hz}$, reflecting the growth of $\ell=2$ mode of SASI with timescales of $O(10) \mathrm{ms}$. Such a feature is found to be generic among $2 \mathrm{D}$ models computed here.

It should be noted that the approximations taken in the simulation, such as the excision inside the PNS with its fixed inner boundary and the light bulb approach with the isentropic luminosity constant with time, are the very first step to model the dynamics of the neutrinoheating explosion aided by SASI and study the resulting GWs. As already mentioned, the excision of the central regions inside PNSs may hinder the efficient gravitational emission of the oscillating neutron star (Ott et al. 2006). It is recently reported that the waveforms obtained in the 2D ray-by-ray Boltzmann simulations (Marek et al. 2009) show a negatively growing feature. At first glance, our results may seem to contradict with theirs. They explained that the negative growth is due to the enhanced neutrino emission of muon and tau neutrinos near the equator of the PNS observed in their simulations. As we mentioned repeatedly, such feature inside the PNSs, cannot be captured here in principle. Outside the PNSs, however, the stronger neutrino emission of $\nu_{e}$ and $\bar{\nu}_{e}$ along the polar regions are also 
seen in their simulations (see their bottom right panel of Figure 11 outside of $30 \sim 40 \mathrm{~km}$, which is the surface of the PNS guessed from their Figure 1), which we can say, consistent with our results. As illuminated by this study, the elaborate estimation of the neutrino anisotropy is crucial to understand the gravitational radiation in supernova cores. This naturally needs a full transport simulation coupled to 3D hydrodynamics encompassing the whole iron core, which is beyond the scope of this study and a grand challenge for all the supernova modellers.

In addition, one more major deficit is the axial symmetry assumed in the present 2D simulations. In $3 \mathrm{D}$, the pronounced dominance of $l=1,2$ along the symmetry axis, which is a coordinate singularity in the $2 \mathrm{D}$ computations, may become weaker, owing to the additional spatial degree of freedom in the azimuthal direction. In the $3 \mathrm{D}$ case, we expect that the amplitudes become smaller owing to the reduced anisotropy along the symmetry axis (Iwakami et al. 2008a, b, 2009). Thus the amplitudes calculated in this study could be an upper bound, in which the maximal anisotropy generated by SASI could be achieved. With the ray-tracing method and the GW formulae derived here, we are now able to investigate the properties of the cross mode of GWs (e.g., Kotake et al. (2009)). We think it important because they are of genuine 3D origin, which in combination of the plus modes, might give us some hints about the explosion asymmetry. Magnetic effects not only on the growth of SASI (Endeve et al. 2008), but also on the GW waveforms (Kotake et al. 2004) should be interesting. This study is an appetizer before the forthcoming 3D studies to clarify those aspects, which will be presented elsewhere soon.

K.K. expresses thanks to K. Sato for continuing encouragements and also to Yosuke Ogino for informative help. We thank an anonymous referee for careful reading and a long list of useful comments. Numerical computations were in part carried on XT4 and general common use computer system at the center for Computational Astrophysics, CfCA, the National Astronomical Observatory of Japan. This study was supported in part by the Grants-in-Aid for the Scientific Research from the Ministry of Education, Science and Culture of Japan (Nos. 19540309 and 20740150) and Grant-in-Aid for the 21st century COE program "Holistic Research and Education Center for Physics of Self-organizing Systems". 



Fig. 9.- Various properties obtained by the ray-tracing calculation at $t=294 \mathrm{~ms}$ for model A. Right panels show the rays (colored by the value of $f_{\nu}$ for $\epsilon_{\nu}=14 \mathrm{MeV}$ ), which are 

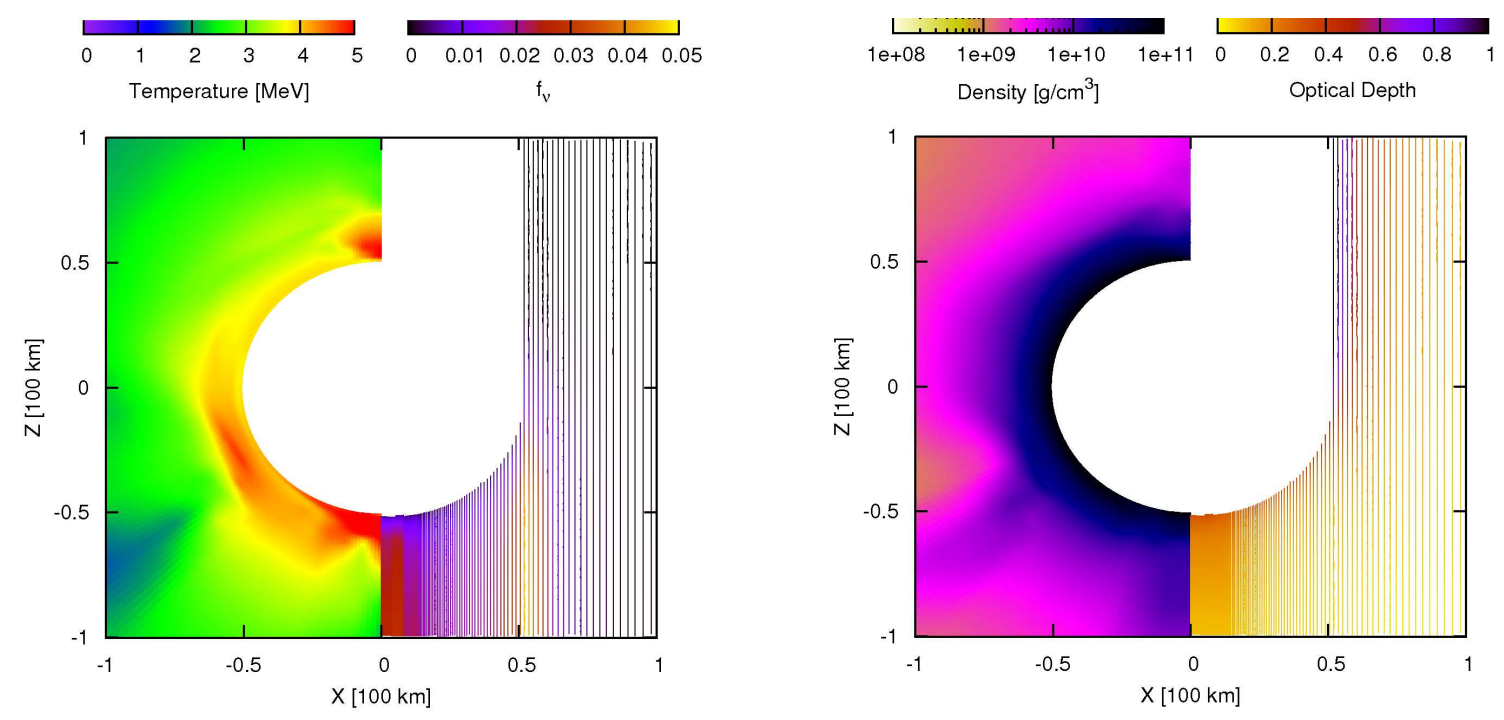

Fig. 10.- Left panel is an enlargement of the bottom right panel of Figure 9 near the central PNS (white circle). Right panel depicts the optical depth of neutrinos at $\epsilon_{\nu}=14$ $\mathrm{MeV}$ (right-hand side) with the density distributions (left-hand side). The opacity sources are taken to be the neutrino emission/absorption as mentioned in section 2.2. It can be seen that high values of $f_{\nu}$ come from the high temperature regions in the vicinity of the PNS, where are thin to neutrinos with the optical depth being smaller than $2 / 3$.
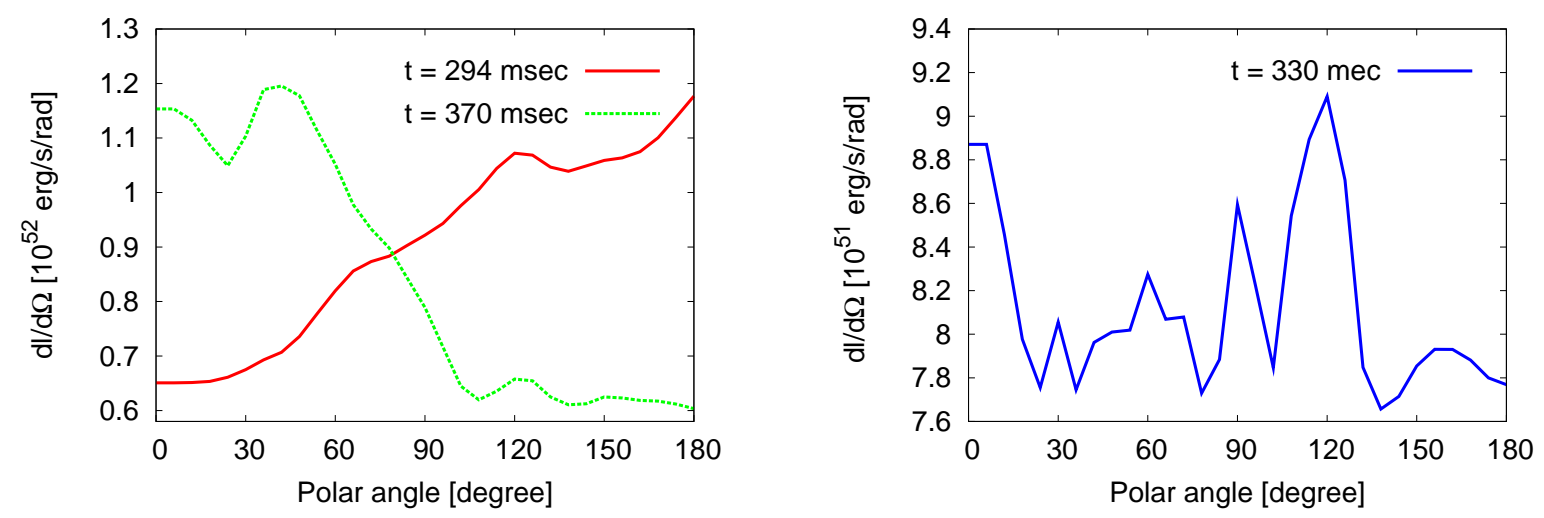

Fig. 11. - The directional dependent neutrino luminosities of $d l_{\nu} / d \Omega$ as a function of the polar angle for model A (left panel) at $t=294$ and $t=370 \mathrm{~ms}$, and model B (right panel) at $t=330 \mathrm{~ms}$. 

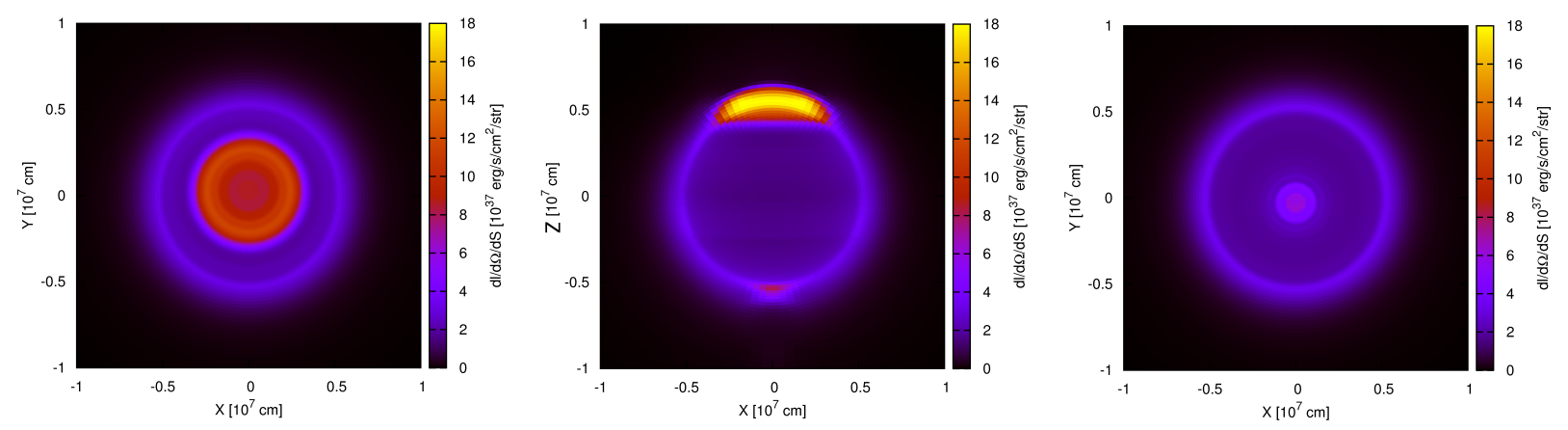

Fig. 12. - Neutrino energy fluxes of $d l_{\nu} /(d \Omega d S$ ) (equation(13) ) of model $\mathrm{A}$ at $t=370 \mathrm{~ms}$, seen from the northern hemisphere (left), the equator (middle), and the southern hemisphere (right), respectively.

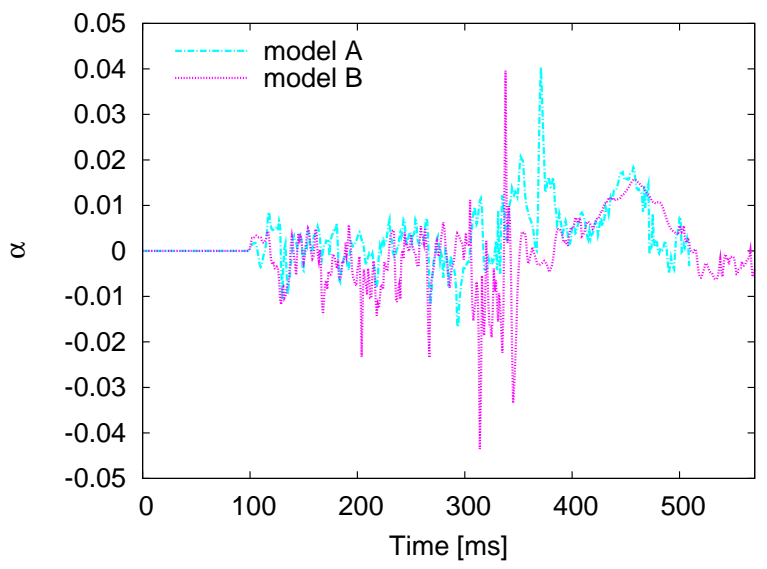

Fig. 13. - Time evolution of the neutrino anisotropy parameter $(: \alpha$ in equation (15) $)$ for models A and B. $\alpha$ keeps positive value with time in the later phase $(\gtrsim 400 \mathrm{~ms})$ when the low-modes explosion is triggered by SASI along the symmetry axis. 

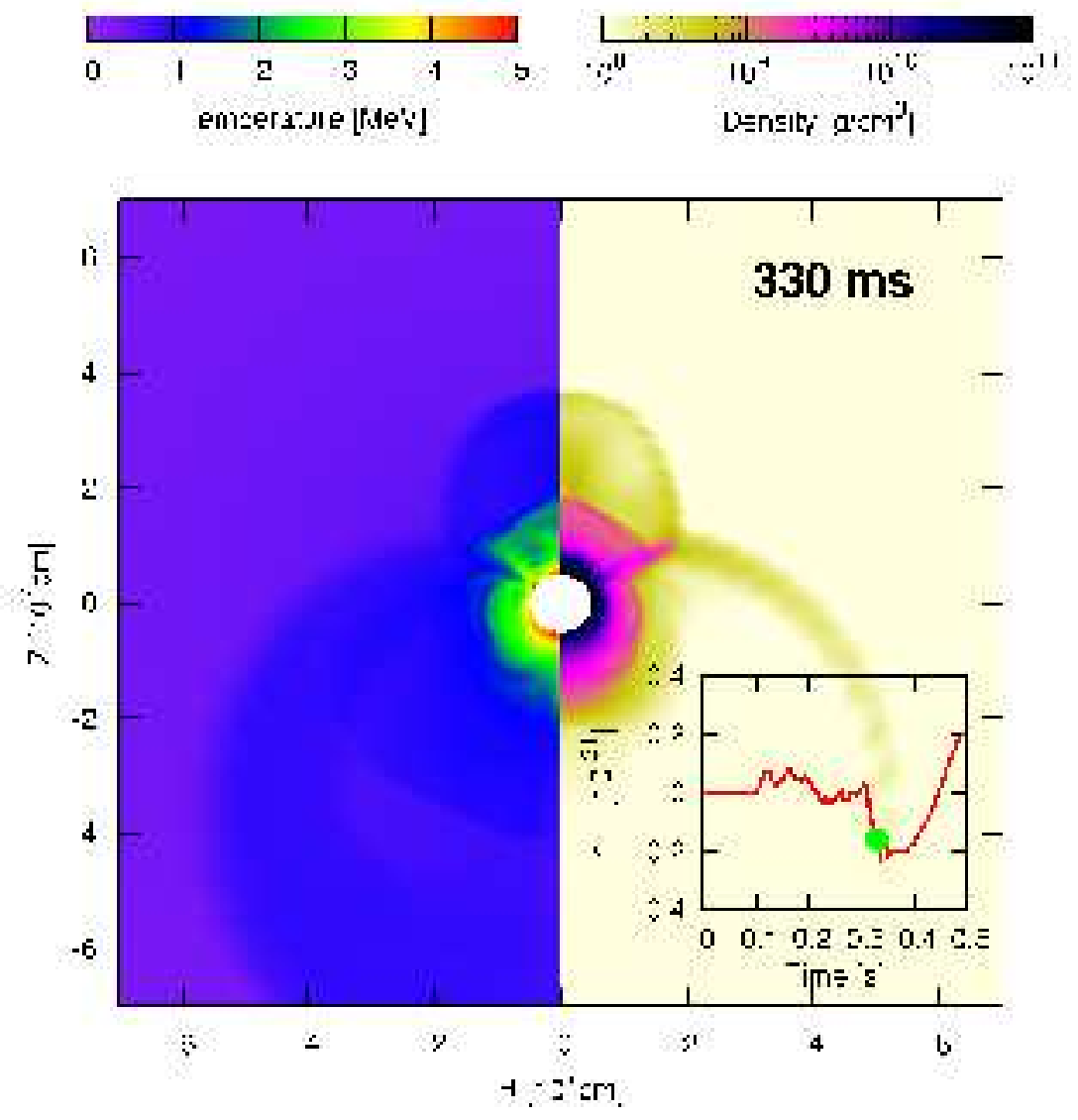

Fig. 14.- Same as Figure 8 but for model B at $t=330 \mathrm{~ms}$.
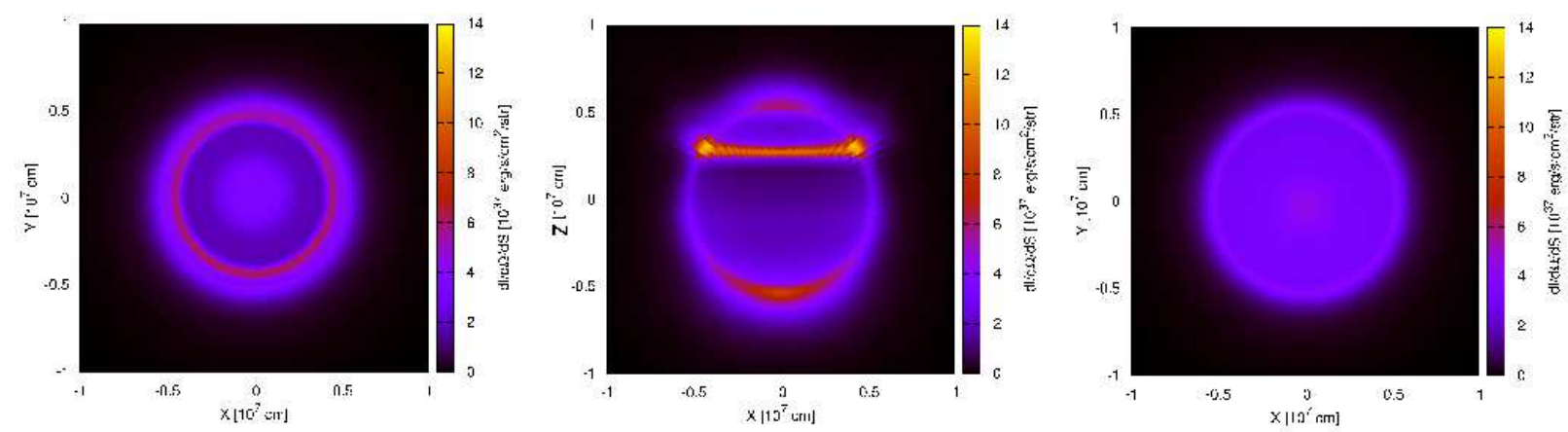

Fig. 15.- Same as Figure 12 but for model B at $t=330 \mathrm{~ms}$, showing the stronger emission seen from the equator (middle), as opposed to the cases of Figures 9 and 12 . 


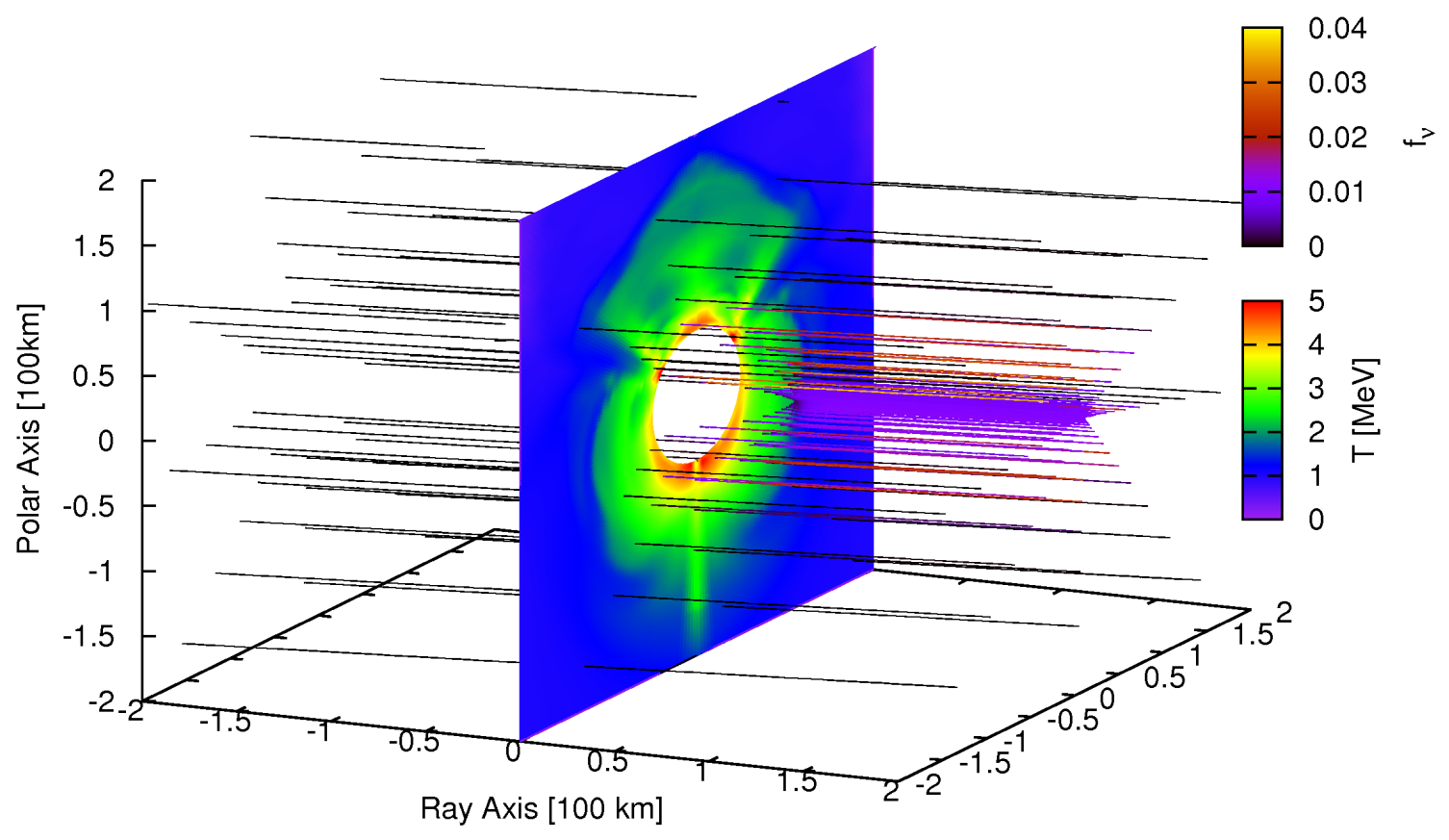

Fig. 16. - Same as Figure 9 (middle right) but for model B at $t=330 \mathrm{~ms}$. 


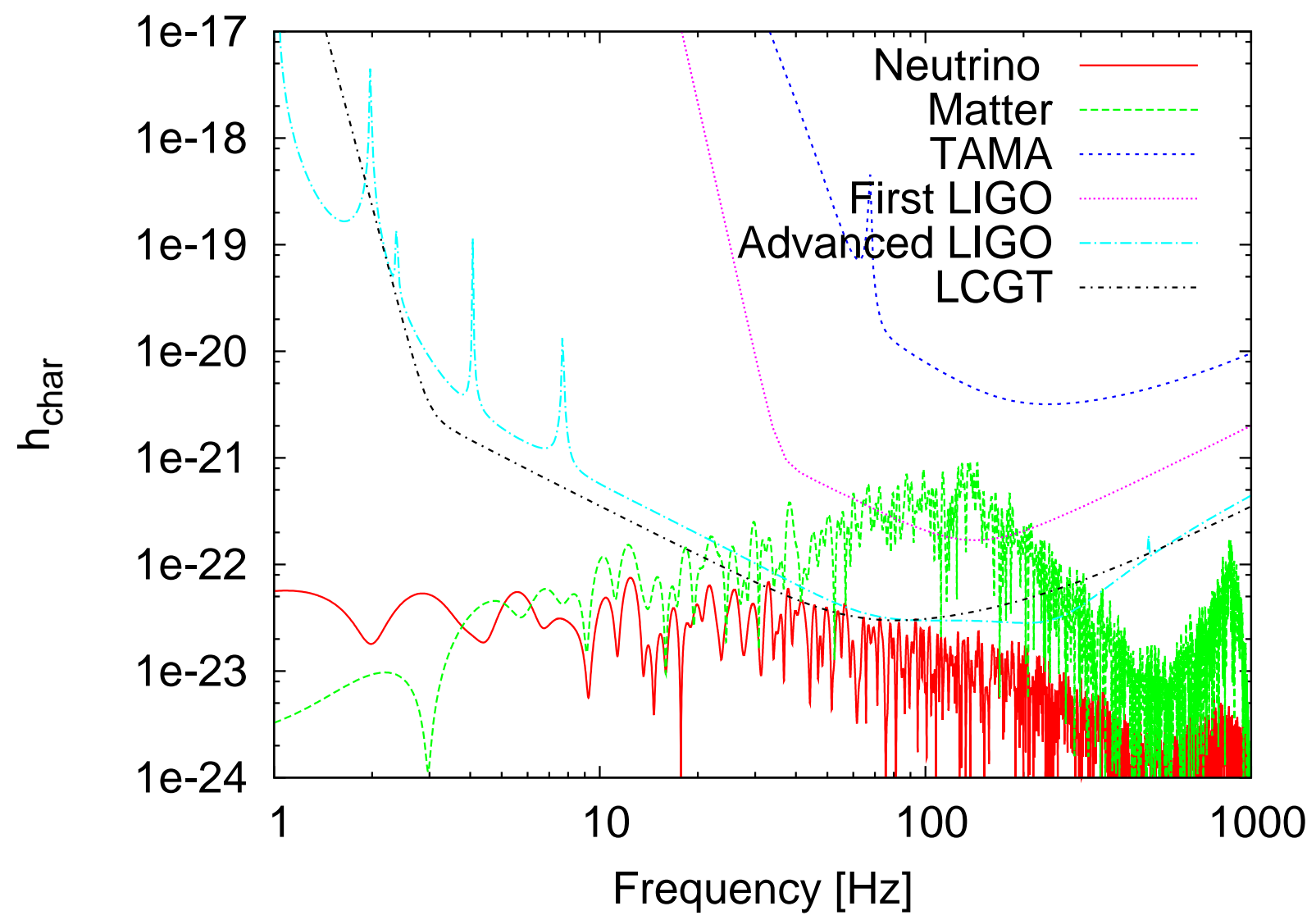

Fig. 17. - Spectral distributions of GWs from matter motions (Matter) and anisotropic neutrino emission (Neutrino) for model A with the expected detection limits of TAMA (Ando \& the TAMA collaboration 2002), first LIGO (Thorne 1995), advanced LIGO (Weinstein 2002), and Large-scale Cryogenic Gravitational wave Telescope (LCGT) (LCGT Collaboration 1999). $h_{\text {char }}$ is the characteristic gravitational wave strain defined in Flanagan \& Hughes (1998). Note that the supernova is assumed to be located at the distance of $10 \mathrm{kpc}$. 


\section{REFERENCES}

Abbott, B., et al. 2005, Phys. Rev. D, 72, 122004

Ando, M., \& the TAMA collaboration 2002, Classical and Quantum Gravity, 19, 1409

Ando, M., et al. 2005, Classical and Quantum Gravity, 22, 1283

Ardeljan, N. V., Bisnovatyi-Kogan, G. S., \& Moiseenko, S. G. 2005, MNRAS, 359, 333

Bethe, H. A. 1990, Reviews of Modern Physics, 62, 801

Blondin, J. M., Mezzacappa, A., \& DeMarino, C. 2003, ApJ, 584, 971

Blondin, J. M., \& Mezzacappa, A. 2007, Nature, 445, 58

Braginskii, V. B., \& Thorne, K. S. 1987, Nature, 327, 123

Buras, R., Rampp, M., Janka, H.-T., \& Kifonidis, K. 2003, Physical Review Letters, 90, 241101

Burrows, A. \& Hayes, J. 1996, Physical Review Letters, 76, 352

Burrows, A., Livne, E., Dessart, L., Ott, C. D., \& Murphy, J. 2006, ApJ, 640, 878

Burrows, A., Dessart, L., Livne, E., Ott, C. D., \& Murphy, J. 2007, ApJ, 664, 416

Bruenn, S. W. 1985, ApJS, 58, 771

Cerdá-Durán, P., Font, J. A., \& Dimmelmeier, H. 2007, A\&A, 474, 169

Dimmelmeier, H., Ott, C. D., Janka, H.-T., Marek, A., Müller, E. 2007, Physical Review Letters, 98, 251101

Dimmelmeier, H., Ott, C. D., Marek, A., \& Janka, H.-T. 2008, Phys. Rev. D, 78, 064056

Endeve, E., Cardall, C. Y., Budiardja, R. D., \& Mezzacappa, A. 2008, arXiv:0811.3385

Epstein, R. 1978, ApJ, 223, 1037

Favata, M. 2008, arXiv:0812.0069

Flanagan, É. É., \& Hughes, S. A. 1998, Phys. Rev. D, 57, 4566

Foglizzo, T., Galletti, P., Scheck, L., \& Janka, H.-T. 2007, ApJ, 654, 1006

Fryer, C. L. 2004, ApJ, 601, L175 
Fryer, C. L., Holz, D. E., \& Hughes, S. A. 2004, ApJ, 609, 288

Heger, A., Woosley, S. E., \& Spruit, H. C. 2005, ApJ, 626, 350

Hiramatsu, T., Kotake, K., Kudoh, H., \& Taruya, A. 2005, MNRAS, 364, 1063

Hough, J., Rowan, S., \& Sathyaprakash, B. S. 2005, Journal of Physics B Atomic Molecular Physics, 38, 497

Iwakami, W., Kotake, K., Ohnishi, N., Yamada, S., \& Sawada, K. 2008, ApJ, 678, 1207

Iwakami, W., Kotake, K., Ohnishi, N., Yamada, S., \& Sawada, K. 2008, arXiv:0811.0651, accepted by ApJ

Iwakami, W., Ohnishi, N., Kotake, K., Yamada, S., \& Sawada, K. 2009, Ap\&SS, 322, 43

Janka, H.-T., \& Mueller, E. 1996, A\&A, 306, 167

Kifonidis, K., Plewa, T., Scheck, L., Janka, H.-T., Müller, E. 2006, A\&A, 453, 661

Kotake, K., Yamada, S., \& Sato, K. 2003, ApJ, 595, 304

Kotake, K., Yamada, S., \& Sato, K. 2003, Phys. Rev. D, 68, 044023

Kotake, K., Sawai, H., Yamada, S., \& Sato, K. 2004, ApJ, 608, 391

Kotake, K., Yamada, S., Sato, K., Sumiyoshi, K., Ono, H., \& Suzuki, H. 2004, Phys. Rev. D, 69, 124004

Kotake, K., Sato, K., \& Takahashi, K. 2006, Reports of Progress in Physics, 69, 971

Kotake, K., Ohnishi, N., \& Yamada, S. 2007, ApJ, 655, 406

Kotake, K., Iwakami, W., Ohnishi, N., \& Yamada, S. 2009, ApJ, 697, L133

LCGT Collaboration, Int. J. Mod. Phys. D, 5, 557, (1999).

Marek, A., \& Janka, H.-T. 2009, ApJ, 694, 664

Marek, A., Janka, H.-T., Müller, E. 2009, A\&A, 496, 475

Mönchmeyer, R., Schaefer, G., Mueller, E., \& Kates, R. E. 1991, A\&A, 246, 417

Müller, E., Rampp, M., Buras, R., Janka, H.-T., \& Shoemaker, D. H. 2004, ApJ, 603, 221

Mueller, E., \& Janka, H.-T. 1997, A\&A, 317, 140 
Obergaulinger, M., Aloy, M. A., Müller, E. 2006, A\&A, 450, 1107

Ohnishi, N., Kotake, K., \& Yamada, S. 2006, ApJ, 641, 1018

Ohnishi, N., Kotake, K., \& Yamada, S. 2007, ApJ, 667, 375

Ott, C. D., Burrows, A., Livne, E., \& Walder, R. 2004, ApJ, 600, 834

Ott, C. D., Burrows, A., Thompson, T. A., Livne, E., \& Walder, R. 2006, ApJS, 164, 130

Ott, C. D., Burrows, A., Dessart, L., \& Livne, E. 2006, Physical Review Letters, 96, 201102

Ott, C. D., Burrows, A., Dessart, L., \& Livne, E. 2008, ApJ, 685, 1069

Ott, C. D 2009, Classical and Quantum Gravity, 26, 063001

Scheck, L., Plewa, T., Janka, H.-T., Kifonidis, K., Müller, E. 2004, Physical Review Letters, 92, 011103

Scheck, L., Kifonidis, K., Janka, H.-T., Müller, E. 2006, A\&A, 457, 963

Scheidegger, S., Fischer, T., Whitehouse, S. C., \& Liebendörfer, M. 2008, A\&A, 490, 231

Shibata, M., \& Sekiguchi, Y. 2004, Phys. Rev. D, 69, 084024

Shibata, M., Liu, Y. T., Shapiro, S. L., \& Stephens, B. C. 2006, Phys. Rev. D, 74, 104026

Stone, J. M. \& Norman, M. L. 1992, ApJS, 80, 753

Suwa, Y., Takiwaki, T., Kotake, K., \& Sato, K. 2007, ApJ, 665, L43

Suwa, Y., Takiwaki, T., Kotake, K., \& Sato, K. 2007, PASJ, 59, 771

Suwa, Y., \& Murase, K. 2009, arXiv:0906.3833

Takiwaki, T., Kotake, K., \& Sato, K. 2009, ApJ, 691, 1360

K.S. Thorne, Gravitational Waves. In Proceedings of the Snowmass 95 Summer Study on Particle and Nuclear Astrophysics and Cosmology, World Scientific, pp. 398-425, (1995).

Walder, R., Burrows, A., Ott, C. D., Livne, E., Lichtenstadt, I., \& Jarrah, M. 2005, ApJ, 626,317

Weinstein, A. 2002, Classical and Quantum Gravity, 19, 1575

Woosley, S. E., \& Heger, A. 2006, ApJ, 637, 914 
Yamada, S. \& Sawai, H. 2004, ApJ, 608, 907

Yamasaki, T., \& Yamada, S. 2005, ApJ, 623, 1000

Zwerger, T., \& Mueller, E. 1997, A\&A, 320, 209 